répondre à une question ansi posée En cffet, les frais de constutution sont doubles; ils comprennent premièrement les honoraires du notane, et secondement les frais d'enregistremont. Les frais de notare sont toujours basés sur le capital. A Paris, par exemple, ils sont de $050 \%$ de I fr. à 500000 francs et de $0.25 \%$ de 500.000 francs à un million. Dans les villes de province, le tanf est moins élevé, 11 est de $050 \%$ de I fr. à 200000 francs, de $025 \%$ de 200.000 francs 500000 francs et de $0.125 \%$ au-dessus.

C'est donc facule à calculer, même sans savori quel est l'objet de la société, puisqu'il suffit de connaître le montant du capilal social.

Mais la deuxième catégorie des frais en matière d'enregistrement n'est pas susceptible de fixation, si l'on ne connaît pas d'une façon très exacte certains détails indispensables :

a) La société ne comprend-elle aucun apport, en d'autres termes est-ellc seulemant une société d'espèces, une réunion de capitaux versés par les actionnaires? Dans ce cas, l'enregistrement ne perçort que $0.25 \%$ sur le montant du capital

b) La société comprend-elle des apports? Dans ce cas, il faut cncore préciser : Si les apports sont rémunérés en actions d'apport sans somme d'argent versé, l'enregistrement est de $0.25 \%$ sur le capital total.

$\mathrm{Si}$, iu contraire, l'apport est rémunéré en argent, ou partie arcent, partie actions, l'enregistrement considere avec raison qu'il y a cession à prix d'argent, un marché à tutre onéreux, et 1 applique le tarif afférent à chaque cession. Si c'est une vente mobilière, le tarif sera de $2 \%$ sur la partie du prix payé en argent; si c'est unc vente immobilière, le fisc exigera le $7 \%$; si c'est la cession d'un bail, ce sera le droit de $0.20 \%$ sur le montant cumulé de toutes les annuités du ball à courir. En un mot, il est impossible de donner une règle formelle, les applications des différents tarifs dépendant évidemment des combinaisons que chacun se propose d'effectuer.

Paul Bougaul, avocat à la Cour d'A ppel de Lyon.

\section{LE LABORATOIRE D'ESSAIS DU CONGERVATORE NATIONAL DES ARTS ET MÉTIERS}

\section{Section des Essais de Machines}

Le Laboratoire d'Essais du Conservatoire National des Arts et Métiers, créé par le décret du I9 mai Igoo, se divise en cinq sections, savoir :

La Section I qui a pour objet les essais physiques.

La Section II qui s'occupe des essais des métaux.

La Section III des essais des matériaux de construction.

La Section $I V$ des essais des machines.

La Section $V$ dont l'objet principal concerne les recherches ou essais sur les matières végétales nouvelles ou insuffsamment connues.

Nous nous proposons aujourd'hui de décrire la section des essais de machines, en passant rapidement en revue ses différents services et son outillage, et en nous arrêtant plus longuement sur certains appareils nouveaux, ou particulièrement intéressants.

Les services de la section des machines peuvent se diviser en trois groupes, occupants des locaux séparés qui s'étendent environ sur une surface de mille mètres carrés.

Le premier groupe occupe une ancienne usine à vapeur, qui servait autrefois à l'éclairage du Conservatoire,principalement des amphithéatres; cette usine a été transformée et aménagée pour répondre au essais de machines à exécuter.
Le deuxième groupe est affecté aux essais des machines hydrauliques : pompes, turbines, compteurs d'eau, (etc., (1) occupe le sous-sol d'un ancien bâtiment du Conservatoire, dit sous-sol Vaucanson).

Enfin le troisième groupe, qui occupe la moitié de la grande salle des nouveaux locaux du laboratoire, dans lequel se font tous les essais de machines therniques autres que la machine à vapeur, et les essais de toutes les machines ne rentrant pas dans les deux groupes précédents.

Comme on le verra dans la suite par la description détail. lée de ces différents groupes, notre installation offre aux industriels les ressources suffisantes pour l'exécution des essais les plus importants. Pour son organisation, nous nous sommes inspirés des beaux laboratoires installés à l'étranger. et que nous avons eu la bonne fortune, au moins pour quelques-uns d'éntre eux, de visiter en détail.

Le laboratoire de Zurich, celui de Berlin installé à Charlottenbourg, et enfin celui de la ville de Dresde, dont la section des machines organisée par le professeur Mollier et celle de l'hydraulique par le professeur Levicki, sont des merveilles du genre; on jugera de l'importance de ce laboratoire quand nous aurons dit que, pour ces deux installations des essais de machines et des essais hydrauliques, on a dépensé 2.500 .000 marks, et que la plupart des constructeurs ont donné leurs machines gratuitement ou, tout au moins, à des prix bien inférieurs aux prix de revient.

Il est vrai que ces laboratoires ont été créés, non seulement pour l'exécution, des essais industricls, mais aussi pour l'instruction des élèves et la formation des ingćnieurs; chez nous, notre organisation vise seulement l'exécution des recherches ou études, et des essais industriels payants.

\section{I. - GROUPE DES ESSAIS DE MACHINES A VAPEUR}

L'ancienne usine d'éclairage électrique du Conservatoire a été entièrement transformée pour exécuter les essais des machines à vapeur légères ou facilement transportables, les essais des machines lourdes et encombrantes ayant lieu chez les industriels ou nous transportons les appareils de mesures nécessaires à l'organisation de ces essais.

Cette salle se divise en trois locaux séparés. Dans le premier est installée une chaudière Belleville, qui peut débiter environ goo kgs. de vapeur à l'heure, à I 2 kilogrammes de pression; elle est alimentée par un petit cheval qui puise l'eau d'alimentation dans des baches graduées et jaugées permettant de mesurer avec précision la vapeur produite par le générateur.

Dans nos essals de vaporisation, nous déterminons la siccité de la vapeur (eau de primıge), à l'aide de l'appareil très ingénieux imaginé par $M$. Rateau, le savant mécanicien bien connu. Cet appareil de mesure présentant un véritabic interêt, nous en donnerons ici la description détaillée.

Appareil de M. Rateau pour mesurer l'eau de primage. - Ala prise de vapeurdu générateur et, le plus près possible de la vanne de sortie de la chaudière, nous avons disposé un bout de conduite $\mathbf{A}$ destiné à prélever l'échantillon de vapeur à analyser, et à porter l'appareil en question.

Cet appareil de mesure se compose d'une conduite bifur* quée $r$, partageant en deux parties égales l'échantillon de prise; l'une de ces parties se rend directement par $\mathrm{C}$ dans une petite chambre $M$, appelée mélangeur, tandis que la seconde partie n'y arrive qu'après s'être convenablement surchauffée par son passage dans un serpentin B, placé dans un fourneau alimenté au gaz de ville, ou par tout autre combustible. Cot appareil est schématiquement représenté 
par la figure $\mathrm{I}$ ci-jointe, sur laquelle $\mathrm{P}$ est une prise pour placcr un manomètre étalon, et $\mathrm{R}$ un robinet à pointeau pour régler le débit de la vapeur.

De la mesure des températures aux différents points du parcours de la vapeur on déduit la quantité d'eau entraînée par les formules établies plus loin.

On prend la température $t_{2}$ du mélange dans le mélangeur, la température $t_{1}$ de la vapeur surchauffée au moment où elle entre dans le mélangeur, et enfin la température $t$ de la prise d'échantillon par la pression de Ja vapeur expérimentée. Soit dans ces conditions:

$t$ la température initiale de la vapear.

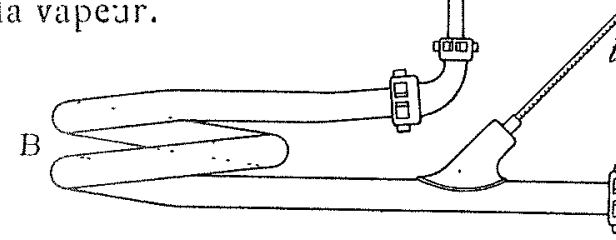

$0_{1}$ la surchauffe donnée à la première partic de la vapeur. $0_{2}$ la surchauffe finale du mélange.

$k$ la proportion de vapeur passant dans le réchauffeur.

$(I-k)$ la proportion ne passant pas dans le réchauffeur.

Le poids $k$ de vapeur surchauffée passe dans le mélangeur de la température $t+0_{1}$ à la température $t+0_{2}$, il perd une quantité de chaleur.

$$
\approx \times k\left(\theta_{1}-g_{2}\right)
$$

$\alpha$ étant la chaleur spécifique de la vapeur d'eau pour une température voisine de $t$.

Le poids $(t-k)$ de vapeur à $t$ degrès, qui arrive directement dans le mélangeur, contient un poids $(\mathrm{r}-k) x$ d'eau. Cette eau est d'abord vaporisée à $t$ degrés, elle absorbe $l(\mathrm{I}-l) x, l$ étant la chaleur latente de vaporisation de l'eau à $t^{\circ}$, puis le poids de vapeur $(\mathrm{I}-T)$ est élèvé de $t$ à $t+\theta_{2}$ en absorbant une quantité de vapeur $\alpha(\mathrm{I}-l i) 0_{2}$, la chaleur totale absorbée est donc :

$$
l(1-k) \dot{x}+a(I-k) 0_{2} \text {. }
$$

En égalant (1) à (2) on a la relation qui donne $x$,

$$
x=\frac{\alpha}{l} \times \frac{k 0_{1}-0_{9}}{1-k}
$$

Si l'on a convenablement construit l'appareil pour avoir $k=\mathrm{I}-k$, ou $k=1 / 2$, la relation (3) devient :

$$
x=\frac{\alpha}{l}\left(0_{1}-20_{2}\right)
$$

$0_{1}$ est donné par la différence $t_{1}-t$ et $\theta_{2}$ par la différence $t_{2}-t$.

La valeur de $k$ est mesurée expérimentalement par condensation de la vapeur à l'extrémité de l'appareil, après le mélangeur, en bouchant alternativement les conduites $C$ et B pour différentes ouvertures du robinet doseur.

La formule donnant la valeur de $x$ se simplifie beaucoup dans le cas où l'on opère sur une chaudière fonctionnant au régime de $5 \mathrm{kgs}$. par centimètre carré, en effet, on a dans ces conditions :

$$
t=150^{\circ} \quad l=500 \quad \alpha=0,5
$$

la valeur de $x$ devient alors, toujours pour $k=\frac{1}{2}$ :

$$
x=\text { о, оо г }\left(\theta_{1}-2 \theta_{2}\right)
$$

On peut exprimer la formule en ces termes : "La siccité de la vapeur, exprimée en millièmes, est égale à l'cxci. de la surchauffe de la vaveur à la sortie du réchauffeur. sur le double de la surchauffe du mélange.

Il est à noter que, dans l'emploi de l'appareil, il est bon de s'attacher à réaliser une surchauffe finale de $0_{2}=10^{\circ}$ environ.

Dans le deuxième local, attenant au précédent, et situé derrière la chaudière, est installé un surchauifcur Schwœrer capable de surchauffer 6 à 700 kilogrammes de vapeur à l'heure. Il est disposé de manière à utiliser les flammes perdues de la chaudière, il peut également fonctionner avec son propre foyer, ou encore avec les deux dispositifs réunis, et cela au moyen de registres convenablement disposés dans les carneaux qui conduisent à la cheminée de l'usine les produits de la combustion de ces deux appareils.

Le troisième local constitue la salle d'essais proprement dite. Elle reçoit la vapeur du surchauffeur, ou de la chaudière, dans une grande bouteille séparatrice de l'eau entraînée, d'où elle est distribuée par des canalisations munies de vannes convenables aux deux plateformes d'essais des machines.

Une machine Weyher et Richemond compound, horizontale demi-fixe, occupe une partie de cette salle; clle peut développer 5o chevaux, et actionner une transmission générale sur laquelle sont disposées des poulies fixes, folles ou à embrayage mécanique, permettant d'actionner tout appareil mécanique à soumettre aux essais; un frein à circulation d'eau est monté sur l'autre arbre de transmission. Cette machine peut fonctionner sur un condenseur par mélange, ou sur un condenseur par surface.

Dans cette salle sont installées 3 plateformes d'essais, qui reçoivent d'un côté la vapeur, et de l'autre côté sont en connexion avec la conduite d'un condenseur par surface pouvant condenser $600 \mathrm{kgs}$ de vapeur à l'heure; une conduite spéciale permet également d'échapper à l'atmosphère la vapeur des machines fonctionnant à échappement libre.

Le condenseur par surface, fourni par la maison Delaunay-Belleville, comporte une pompe, mise en action par un moteur électrique, qui déverse l'eau condensée dans deux bàches graduées permettant de mesurer avec précision la vapeur condensée des machines en essai. Ce condenseur est également mis, par une conduite spéciale, en communıcation directe avec la bouteille de vapéur. Le condenscur par mélanges est du type Weyher et Richemond.

L'eau de refrigération de ces deux appareils est empruntée aux canalisations de la ville.

Enfin, dans le cas où la vapeur fournie par la chaudière dont nous venons de parler est insuffisante, il existe, dans un sous-sol attenant à cette installation, une batteric de 4 générateurs Belleville qui peuvent nous donner $5.000 \mathrm{kgs}$ environ de vapeur à l'heure; ces générateurs servent en hiver au chauffage général du Conservatoire. Une conduite spéciale fait communiquer ces chaudières avec la bouteille de vapeur de la salle des essais de machines. La vapeur consommée peut se mesurer sur les bâches d'alimentation, ou, plus simplement encore, à l'aide d'un compteur de vapeur, installé sur cette conduite, et que nous allons décrire maintenant:

Compteur de vapeur. - Cet appareil de mesure est basé sur la vitesse d'écoulement de la vapeur à travers un orifice en mince paroi, en utilisant les formules expérimen- 
tales établies par les très belles expériences de M. Rateau, et publiées dans les Annales des Mines de janvier 1902.

La formule de M. Rateau est :

$$
I_{m}=P(15,26-0,96 \mathrm{lg} . P)
$$

dans laquelle $P$ est la pression en amont de l'orifice, et $I_{m}$ ce que M. Rateau appelle le débit maximum, qu a besoin, dans le cas de mince paroi, d'être corrigé, en tenant compte de la pression aval $p$. - On trouve pour cela, dans le mémoire de $M$. Rateau, cité plus haut, une courbe donnant les valeurs de $\frac{I}{I_{m}}$ en fonction du rapport des pressions $\frac{p}{P}$ portées en abcisses. Dans ces conditions, $I$ correspond au débit réel de l'orifice

$$
I=K \times P(15,26-0,96 \lg P)
$$

$K$ étant égal à $\frac{I}{I_{m}}$ et donné par la table ci-jointe, ou par

la courbe dont nous venons de parler.

Il faut donc connaître la pression amont $P$, ou la pression aval $p$, et la différence de ces pressions $(P-p)$; c'est dans ce but que nous avons créé les appareils suivants.

Le compteur de vapeur se compose de deux tuyaux réservoirs, d'un diamètre plus grand que la conduite sur même hautcur. Pour cela, leur partie supéricure est enve loppée d'un tube d'un diamètre plus grand, dans lequel of fait circuler un courant d'eau froide.

Nous possédons également un compteur de vapcui analogue portatif, de plus petite dimension, qui, dans cen tains cas, nous a rendu des services précieux; par exempli lorsqu'il s'agit, dans un essai de vaporisation, de mesurer ha vapeur consommée par un souffleur de vapeur branché directement sur le générateur en essai.

L'appareil que nous venons de décrire, et qui est installe à poste fixe dans la salle des machines à vapeur, peut se tarer expérimentalement en recueillant dans le condenseur par surface la vapeur qui le traverse, et que nous mesurons à l'état d'eau condensée dans les bâches graduées qui lui font suite; on fait en même temps des mesures de siccitê de la vapeur à l'aide de l'appareil de M. Rateau, placé sur' la chambre, en amont du compteur de vapeur. Des mesures: analogues, faites sur le compteur de vapeur portatif, nous ont donné,entre les résultats expérimentaux et ceux fournis. par la formule, des écarts insignifiants.

Appareil pour l'essai des calorifuges. - Un apparcil spécial, qui se rattache également à ce groupe, est celui

\begin{tabular}{|c|c|c|c|c|c|c|c|c|c|c|c|c|c|c|}
\hline $\begin{array}{l}\text { Valeurs } \\
\text { de } \frac{1}{I_{m}} \text { ou } K\end{array}$ & 0,05 & 0,1 & 0,15 & 0,20 & 0,25 & 0,30 & 0,35 & 0,40 & 0,45 & 0,50 & 0,55 & 0,60 & 0,65 & 0,7 \\
\hline $\begin{array}{l}\text { Valeurs } \\
\text { de } \frac{p}{p}\end{array}$ & 0,9975 & 0,993 & 0,986 & 0,976 & 0,963 & 0,948 & 0,931 & 0,911 & 0,887 & 0,859 & 0,825 & 0,7875 & 0,742 & 0,691 \\
\hline
\end{tabular}

TABLE pour calculer les debits de vapeur correspondant à 1 centimètre carré đ’orifice

laquelle ils sont placés. Ils sont séparés par un diaphragme percé d'un orifice en mince paroi, d'une dimension telle que la chute de pression $P-p$ ne soit pas trop élevée. Chacun de ces réservoirs $A$ et $B$ est muni d'une conduite de purge, pour éviter l'engorgement de l'appareil par l'eau de condensation, et chacun d'une prise de vapeur conduisant au manomètre différentiel.

Ce manomètre est d'une construction très spéciale; il a été étudié par nous, de concert avec la maison Bourdon. Il se compose d'un tube manométrique ordinaire déplaçant

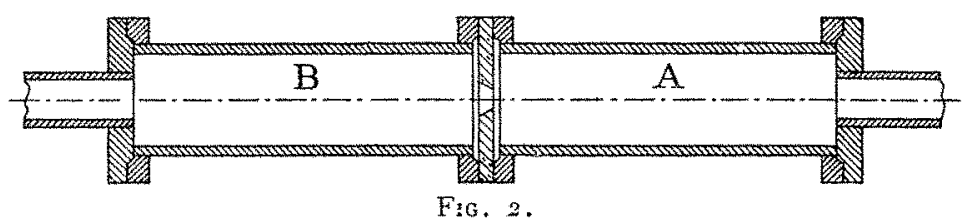

une aiguille sur un cadran; ce tube est mis en communication avec la chambre amont $\mathrm{A}$ du compteur ; ce manomètre est tout entier rentermé dans une boìte très solide dans laquelle règne la pression de la chambre aval $\mathrm{B}$ du compteur. La partie avant de la boîte possède une glace très résistante par laquelle on lit la graduation du manomètre intérieur qui est éclairé à l'aide d'une lampe électrique placée sur la face opposée de la boîte a pression. Cet appareil différentiel est gradué de o à 12 mètres d'eau. Certaines précautions sont à prendre pour que les lectures faites sur le manomètre soient correctes. Comme il s'agit de lire des différences de pression très faibles, il faut purger d'air avec grand soin les deux conduites mettant en connexion le manomètre avec les chambres A et B du compteur; en outre, il faut, par un système de réfrigération convenable, maintenir ces deux conduites pleines d'eau de condensation à la des essais d'enduits calorifuges. Nous allons, en quelques mots, exposer la méthode que nous avons adoptée pour ces essais ( 1 ):

De nombreuses demandes d'essais de matières calorifuges nous ont amené à rechercher un appareil simple et pratique, propre à mesurer leur valeur ou, du moins, à les comparer entre elles.

Nous avons d'abord porté nos recherches du côté d'un appareil à vapeur, de manière à nous rapprocher le plus possible des conditions industrielles dans lesquelles les calorifuges sont généralement employés. Mais des difficultés matérielles nous ont fait abandonner cette voie pour étudier un appareil électrique dans lequel les mesures offrent une facilité et une précision bien supérieures aux autres méthodes.

La méthode employée consiste à porter un élément de conduite à une température $t$ déterminée, que l'on maintient rigoureusement constante pendant le temps nécessaire. On mesure les calories consommées pour maintenir cette température $t$ constante, d'abord pour un élément de conduite non calorifugée, ensuite pour un élément de même dimension recouvert du calorifuge à expérimenter. Le chauffage de l'appareil est obtenu au moyen d'un courant électrique dont le réglage et la mesure sont faciles.

En résumé, nous mesurons $W_{1}$ et $W_{2}$, quantités d'énergie électrique nécessaires pour maintenir cette température $t$ constante dans chacun des deux éléments de conduite.

Deux tubes de bronze, de i mètre de longueur, sur omo 7 de diamètre et $10 \mathrm{~mm}$. d'épaisseur (cette épaisseur a pour objet

(1) L'étude de cet appareil est due à M. Bor Er-Gunlon, chef de section au Laboratorre, et à MM. Auchair et Dusnur, assistants. 
de permettre une mesure plus précise de la température du tube lui-même) sont placés à côté l'un de l'autre, comme le montre la figure 3 ci-jointe.

Chacun d'eux contient intérieurement une résistance électrique $r_{1}, r_{2}$, destinée à le chauffer : c'est un fil de rhéostatine isolé à l'amiante, enroulé très régulièrement sur un tube de fer, dont les extrémités sont fixées dans les bouchons qui obturent les tubes. Le courant entre dans la résistance par une borne isolée. Les tubes $A_{1}$ et $A_{2}$ sont suspendus au plafond par des fils fins et isolants. La température est mesurée dans chaque tube par trois

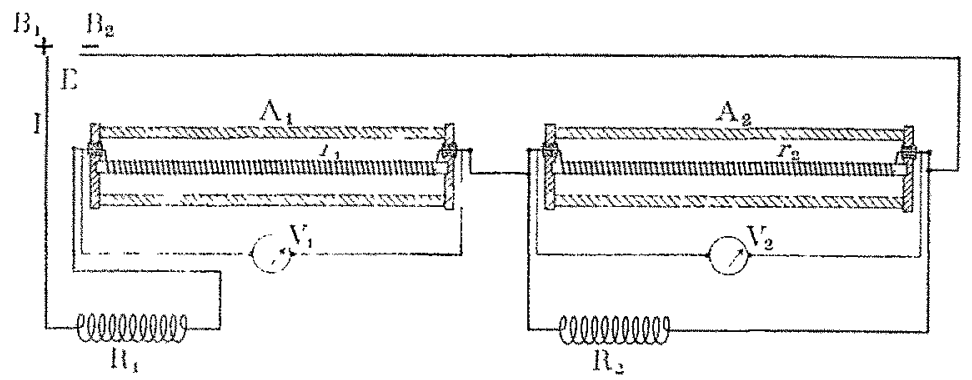

Fig. 3.

thermomètres placés aux extrémités et au milieu; le courant électrique, pris au tableau à, la borne $B_{1}$, à la tension $E$ du secteur, traverse une résistance réglable $\mathrm{R}_{4}$ qui crée une chute de tension $e_{1}$, de manière à obtenir le courant $I$ désirable, puis la résistance intérieure $r_{1}$ du tube $\Lambda_{1}$, et la résistance intérieure $r_{2}$ du tube $A_{2}$ recouvert de calorifuge. Aux extrémités de ce tube est montée en dérivation une résistance $R_{2}$, destinée à régler l'intensité du courant qui traverse $A_{g}$. De là, le courant retourne à la borne $B_{3}$.

Quelquefois, les deux tubes sont montés indépendament l'un de l'autre, ce qui facilite le réglage des températures, mais augmente la dépense d'électricité. Un voltmètre de précision à double direction permet de mesurer la différence de potentiel entre les bornes de $A_{1}$, et celles de $A_{2}$; un anpèremètre Chauvin, également à double direction, mesure les intensités des courants traversant les deux tubes. On a ainsi, quand le régime est établi, les éléments nécessaires pour caractériser le calorifuge.

Nous avons publié les résultats d'un certain nombre d'essais dans le $n^{0} 7$ du Bullelin du Laboratoire, ils constituent une sorte d'échelle, entre les éléments de laquelle viennent se placer les calorifuges que les industriels nous soumettent.

On opère dans une salle dont on mesure la température, qui doit être peu variable. On fait passer le courant et, en manœuvrant convenablement les résistances $R_{1}$ et $R_{2}$, on amène les deux tubes aux mêmes températures. On mesure alors $W_{1}$ et $W_{2}$, quantités de chaleur perdue à $t$ degrés par mètre de tube nu et par mètre de tube recouvert de calorifuge.

On établit alors pour le tube nu et pour le tube calorifugé une courbe donnant les tompératures en fonction des calories perdues par heure.

Nous arons ainsi :

$$
e_{t}=\frac{W_{1}-W_{9}}{W_{i}}
$$

et est ce que nous avons appelé le coefficient d'économie du calorifuge essayé pour l'écart de température $t$. C'est le nombre par lequel il faut multiplier le prix correspondant aux pertes de chaleur d'une canalisation nue pour obtenir l'économie qu'on réalisera en revêtant cette canalisation du calorifuge étudié.
On fait, en général, une série de lectures entre 80 et $330^{\circ}$, sur des tubes horizontaux; 'quelquefois, on les répète sur les mêmes tubes placés verticalement. Pour l'étude complète d'un calorifuge, nous faisons un grand nombre d'essais, de manière à construire la courbe complète caractérisant ce type de calorifuges. C'est ainsi que nous avons opéré pour ceux qui constituent notre échelle de base.

Pour la température des tubes, on prend la moyenne des lectures des trois thermomètres, dont l'écart est très faible. Comme $e_{t}$ varie très lentement avec $t$ (ce fait est vérifié non seulement par nos essais, mais par les expériences de Norton), l'erreur commise en prenant la moyenue des trois lectures est très faible.

La stabilité de la température ne s'obtient qu'après une marche de plusieurs jours. Nos essais nous ont montré : que l'étude du tube nu pouvait être faite une fois pour toutes; que les résultats obtenus avec les tubes horizontaux et verticaux étaient les mêmes; enfin que chaque caloriluge peut être étudié seul, sans opérer en même temps sur le tube nu.

Dans un prochain article nous décrirons le groupe des essais hydrauliques.

\section{(A suivre.)}

Boyer-Gullon, Ingénieur ciril des Mines,

Chef de la section des essais de machnes an Laboratone d'Essats du Conserratore Natoral des Ants et Méthers.

\section{EXPÉRIENCES FAITES PAR MM. WILSON \& CORE SUR DES MODĖLES DE BARRAGE}

L'êtude de laménagoment rationnel des eaux, lant au point de l'alimentation des villes qu'à celui des irrigations, ou de la production de forces motrices, conduit souvent it constituer des réserves d'eau de très grande capacité, ct, par suite, à atablir des harrages de grande hauteur dont la. construclion entraine toujours une dépense considirable. On est alors conduit à serror de très pres la question, de manière à réaliser le profil conduisant au strict minımum de dépense, tout en réalisant une parfarte stabilité. cette dernière condition dominant de haut toutes les autres.

Cette question de la stabilité des barrages préoccupe depuis longtemps les Anglais, dont rimmonse cmpire colonial conlient des régions oil leau fait parfois dufaut de longs espaces de tomps, at dont le développement economique est intimément lié avec la cróation de réservoirs de grande capacité.

A la vérité, la théorie des barrages a été mathómaliqucment mise au point en 1902 par M. Maurice Llevy, dins ses communications sur ce sujet à l'Academie dos Sciences. Toutefois, cette théorie no s'applique qu'au corps proprom ment dil du barrage, et, dans le voisinage de la fondation, cello-ci introduit son influence propre ef modifie, dans une certaine mosure (assez difficile a définir oxactement), la répartition norma'e des efforts developpés à la base. Comme c'est à la base quo les efforts sont los plus considérables, c'est dans cette région qu'il conviendrait de les mieux connaitre; malheureusement, on s'ótail peu inquiélé jusqu'ici de la déterminalion exacte de ce qui devait se passer dans le voisinage de la fondation. It comme cette détermination paraissait devoir ne se prêter que difficilement a un calcul rigoureux, il importait d'avoir recours a l'expérience pour éclaircir celte importante question. «I faut continuellement rappeler notre esprit a l'experience, 